\title{
Género, urbanización y pobreza: el reto de los "hogares"*
}

\author{
Sylvia Chant ${ }^{* *}$ \\ Departamento de Geografía \\ London School of Economics
}

\section{Resumen}

El artículo pone de relieve la importancia de considerar en las intervenciones que busquen alcanzar el desarrollo sustentable las dimensiones de género, urbanización y pobreza y la necesidad de abordarlas desde la perspectiva de los hogares o unidades domésticas, especialmente de aquéllos dirigidos por mujeres.

Al reflexionar acerca del concepto de hogar y al abordar la selectividad por género de la migración rural-urbana, fenómeno que ha influido determinantemente la urbanización en el Tercer Mundo, llama la atención acerca de las estrategias de los hogares del campo y la ciudad que afectan la composición de las unidades domésticas y, resalta la urgencia de considerar a la creciente proporción de hogares pobres dirigidos por mujeres en las ciudades, como objetivos

continúa

- Ponencia elaborada para la Sesión Internacional de Capacitación del Programa LEAD, "Dinámica rural-urbana: intervenciones para el desarrollo sustentable", Okinawa, Jápbn, octubre, 1996.

* Agradezco a las siguientes personas por sus comentarios a una venibn anterior del presente trabajo: doctora Cathy McIlwaine (Queen Mary and Westfield Colleg, Universidad de Londres), doctora Penny Vera-Sanso (Universidad de Kent en Canterbury), Jo Beall (Escuela de Economía de Londres), y David Satterthwaite (Instituto Internacional para el Medio Ambiente y el Desarrollo). 
de políticas de desarrollo sustentable, por ser el hogar una de las instituciones centrales que contribuyen a modelar las relaciones entre hombres y mujeres, su acceso a los recursos y su participación en el cambio para el desarrollo.

Introducción: metas y objetivos

Género, pobreza y urbanización son palabras claves del discurso internacional sobre desarrollo de finales del siglo XX, ${ }^{1} \mathrm{y}$ tienen una importancia particular para la retórica del desarrollo sustentable. Quienes abogan por el "desarrollo sustentable" comparten toda una gama de preocupaciones y enfoques, mientras que aquellos cuyas prioridades son tanto la igualdad social y económica como la protección y la renovación ambientales, hacen hincapié en la necesidad de una participación extensa en la formulación de políticas, la planeación y la forma de gobierno. Esto implica nociones de desarrollo tales como bases, inversión en los individuos, construcción de infraestructura, y permitir un cambio autogestivo $\mathrm{y}$ autocontinuado que tome bajo su responsabilidad el medio ambiente, por medio de vínculos creativos entre agencias multilaterales, dependencias estatales, empresas, organizaciones no gubernamentales (ONG) y grupos comunitarios. Para la puesta en marcha de estas estrategias, en especial si están enfocadas a las desigualdades de género, es de vital importancia reconocer las instituciones sociales que contribuyen a modelar los roles y las relaciones entre hombres y mujeres -mismas que se integran socialmente-, su acceso a los recursos y su participación en el cambio para el desarrollo. Una de estas instituciones es "el hogar", entidad que, de una u otra forma (como se verá más adelante), resulta crítica para la mediación de las acciones, la identidad y las experiencias personales. Sin embargo, "el hogar" representa un

${ }^{1}$ Por ejemplo, todas estas cuestiones han estado presentes, en mayor o menor medida, en as principales conferencias mundiales de los años noventa; en particular: la Conferencia de Naciones Unidas para el Medio Ambiente y el Desarrollo que se celebró en Rio de Janeiro en 1992; la Conferencia Internacional sobre Población y Desarrollo de El Cairo, y la Cumbre Mundial sobre Desarrollo Social de Copenhague, que tuvieron lugar durante 1994; la Cuarta Conferencia Mundial sobre la Mujer, que se llevó a cabo en Beijing en 1995, y la Conferencia HABITAT II, celebrada en Estambul en 1996. conjunto problemático para el pensamiento y la práctica del desarrollo, y con frecuencia se le considera "fuera" del campo de acción de las intervenciones formales, excepto en su acepción casi ubicua como unidad que se integra alrededor del matrimonio monógamo y la procreación, que normalmente asume la forma de una familia nuclear con un jefe masculino y que tal vez, en algunas ocasiones, permite la inclusión de algún familiar adicional. En realidad, el apego ideológico a un estereotipo único y definitivamente eurocéntrico de hogar es tal, que en aquellos casos en que se hace evidente la brecha entre las formas de vida reales e imaginarias de una proporción cada vez mayor de la población mundial, se habla de "descomposición familiar", en particular al referirse al número creciente de hogares encabezados por mujeres. ${ }^{2}$

Sin embargo, la "descomposición familiar ${ }^{\mathrm{n}}$ presupone una norma que no resultaría aplicable para cierto rango de ámbitos sociales y culturales; además, sus matices negativos bien podrían resultar poco apropiados a la luz de las realidades de la vida familiar que transcurre en diferentes tipos de hogares. Asimismo, durante siglos las transformaciones en cuanto a la forma y la organización de los hogares han estado ocurriendo, en todo el mundo, conjuntamente con y como respuesta a cambios socioeconómicos de mayores dimensiones. Por tanto, desde el punto de vista de la práctica del desarrollo, pensar que los hogares son entidades monolíticas, fijas e “inviolables”, puede convertirse en un obstáculo para las intervenciones en materia de alivio de la pobreza urbana y la desigualdad entre géneros. Por otra parte, si se desea que las bases contribuyan de manera integral al desarrollo sustentable, es necesario investigar los hogares de los sectores de bajos ingresos, y saber lo que ocurre en ellos.

Con esto en mente, el objetivo principal de este estudio es desentrañar la importancia que tiene incluir al "hogar" en los análisis sobre la dinámica de la interacción entre génera, urbanización y pobreza. Como parte esencial del presente ejercicio, interesa demostrar el carácter social, y por ende diverso, de los hogares, no sólo a lo largo del tiempo y el espacio, sino dentro de los ámbitos rurales y urbanos contemporáneos. Si bien la flexibilidad y la

${ }^{2}$ Se estima que en la actualidad los hogarescon jefatura femenina representan cera de una quinta parte del total mundial. Aunque parecen tener una larga historia en un gran número de regiones del mundo en desarrollo (en especial en África Subsahariana y el Caribe), y a pesar de que las fuentes de información deficientes hacen difícil establecer las tendencias precisas a lo largo del tiempo, la evidencia de diversos países indica un aumento significativo durante el periodo de posquerra (Chant. 1996b: cap. 3). 
diversidad de la forma que adoptan los hogares con frecuencia ha sido tanto una consecuencia intencionada como una no intencionada del carácter diferenciado según el género del crecimiento urbano y la pobreza en las poblaciones de los países en desarrollo, el apoyo a formas diversas de hogares y vida familiar por parte de los estados, las agencias internacionales y las élites, ha tomado tiempo en concretarse. Esto, a su vez, ha traído consecuencias desfavorables para los individuos de las comunidades de bajos ingresos, particularmente en el caso de miembros de hogares con jefatura femenina. Los argumentos centrales en este estudio refieren que el hecho de no reconocer ni tolerar (sin mencionar la falta de apoyo) la diversidad en los hogares, no sólo debilita las perspectivas de un desarrollo sustentable socialmente integrador, sino que provoca algunos de los "problemas" que los encargados de la formulación e instrumentación de políticas dicen estar enfrentando primordialmente en los entornos urbanos.

Este trabajo se ha dividido en tres secciones principales. La primera se refiere a las cuestiones de género y el proceso de urbanización, y otorga atención especial a la importancia que tienen la organización y las estrategias de los hogares para la estructuración de la selectividad por género de los flujos rnigratorios. Además, se mencionan los impactos que ejerce la movilidad demográfica diferenciada sobre los diversos tipos de hogares que surgen en las áreas urbanas. La segunda sección considera la cuestión de la pobreza urbana, y señala cómo el análisis de las relaciones entre géneros que ocurren dentro de los hogares, así como fuera de ellos, resulta crucial para determinar la calidad y los medios de vida de los sectores de población de bajos ingresos. En la tercera y última parte se hace una referencia breve a ciertos problemas que los encargados de la formulación de políticas y los planificadores interesados en la promoción de un desarrollo socialmente sustentable, podrían abordar para disminuir las desigualdades entre hombres y mujeres. Un gran número de las observaciones que aquí se incluyen se

${ }^{3}$ Un ejemplo podría ser el hecho de que la migración de mujeres o madres solteras se deriva de la falta de atención por parte de las políticas, al acceso de las mujeres a la tierra y a los medios de vida dentro de las comunidades rurales. Otro ejemplo podrían ser los problemasde hacinamiento y de falta de vivienda en las áreas urbanas, dado que los gobiernos y sus dependencias rara vez facilitan a las mujeres la obtención de tierras o viviendas por derecho propio, ya sea dentro de planes de vivienda subsidiados con fondos públicos o por derecho propio, ya sea dentro de planes de vivienda subsidiados con fondos públicos o por 1985).

derivan de experiencias de investigación en México, Costa Rica y Filipinas. ${ }^{4}$ Estas no sólo subrayan la importancia de tomar en cuenta las diversidades tanto intra como interregionales, sino que realzan la necesidad de un análisis contextualizado que sirva de base para las políticas y la planeación participativa eficaces.

\section{Género, hogares y urbanización}

\section{Migración rural-urbana}

Los movimientos diferenciados de hombres y mujeres de las áreas rurales a las urbanas es tal vez uno de los ejemplos más claros de la naturaleza variada según el género de los procesos de urbanización. Los patrones a nivel macro de la migración hacia áreas urbanas del mundo en desarrollo, revelan que en América Latina, el Caribe y el sureste asiático, los flujos migratorios están integrados en su mayoría por mujeres. Por su parte, en África Subsahariana, África del Norte, el Medio Oriente y el sur de Asia, los hombres siguen siendo los principales migrantes. ${ }^{5}$ Mientras que estos patrones entre áreas rurales y urbanas generalmente concuerdan con las variaciones regionales sobre acceso a los recursos económicos conforme al género (tierra, herramientas, empleo, ingreso y otros) (Brydon y Chant, 1989; Chant y Radcliffe, 1992; Townsend y Momsen, 1987), el atractivo que ejercen las generalizaciones no debe cegarnos ante la existencia de dos problemas cruciales que están interrelacionados: primero, que el acceso según género a los recursos está socialmente

4 He realizado investigación sobre género, hogares y pobreza urbana en México desde 1981; desde 1987 en Costa Rica; y desde 1991 en Filipinas. Agradezco a mis entrevistados de campo, a mis colaboradores en diversos proyectos (particularmente la doctora Cathy Macllwaine, quien participó en la investigación sobre Costa Rica y Filipinas), y alas siguiente organizaciones por su apoyo financiero: Consejo para la Investigación Económica y Socia

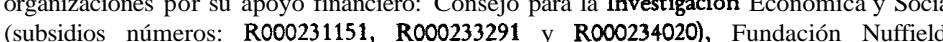
(subsidios números: R000231151, R000233291 y R000234020), Fundación Nuffield Fideicomiso Leverhulme, Academia Britanica, Centro Internacional Suntory Toyota para Londres, Universidad de Liverpool, y Escuela de Economía de Londres.

${ }^{5}$ Debe considerarse que un gran número de hogares con jefatura femenina de África Subsahariana y el sur de Asia, lo son de hecho; los compañeros varones se encuentran Subsahariana y el sur de Asia, lo son de hecho; los compañeros varones se encuentra trabajando en áreas urbanas o en el extranjero, y tienen intención de regresar eventualmente, por lo que aseguran su lugar en las áreas rurales mediante el envío de remesas (Gardner, 1995; Kennedy, 1994). Sin embargo, es importante señalar que en algunos países, como India, la proporción de jefaturas femeninas que representan las viudas es elevada (Baylies, 1996:83 Drèze, 1990 
determinado, y segundo, que la organización de los hogares desempeña una función crítica en la negociación social de los recursos, en la decisión de migrar, y en otros factores. Sin embargo, a pesar de que los hogares (y su organización) no son sujetos fáciles de un tratamiento generalizado, ya sea en términos conceptuales o pragmáticos, la palabra "hogar" casi siempre va precedida por un artículo definido, lo que implica que "el hogar" tiene una forma y significado coherentes y aceptados universalmente.

\section{Conceptuación de "bogar"}

Aunque la mayoría de las fuentes de información nacionales e internacionales clasifican a los hogares como unidades espaciales que se caracterizan por la residencia compartida y la reproducción cotidiana (primordialmente, cocinar y comer), cada vez queda más claro que "el hogar" significa cosas diferentes para diversas personas en distintos lugares. Por ejemplo, en India los hogares pueden entenderse como unidades familiares, unidades económicas, o viviendas (Thorner y Ranadive, 1992:153) y, al igual que en un buen número de países del mundo en desarrollo, las contribuciones de aquellos que viven fuera de las fronteras físicas del hogar bien pueden ser mayores que las de quienes lo habitan (véase Chant, 1996b: cap. I, para discusión y referencias). Como lo resumió un grupo internacional de estudiosos involucrados en el proyecto "Hogares, género y edad" ("Households, Gender and Age") que inició la Universidad de Naciones Unidas a principios de los años ochenta:

El hogar, según sus diferentes connotaciones culturales, es la unidad primaria de vida social. En él están encapsuladas una serie de actividades que desarrollan personas que viven juntas la mayoría del tiempo y que se brindan mutuamente apoyo físico, la organización y el medio más amplios de la comunidad (Masini, 1991:4).

Quiénes son los miembros del "hogar", es también una cuestión difícil de abordar cuando esto cambia varias veces en un solo año (Fonseca, 1991:13), dando lugar así, en cualquier momento, a una variedad extensa de tipos de hogar. Por otra parte, aunque la mayoría de los hogares se basa en lazos de consanguinidad o matrimonio, esto no impide la existencia de unidades que incluyen

amigos, compañeros de trabajo, aprendices, y otras personas. En resumen, los hogares son, y en realidad siempre han sido, diversos en cuanto a su composición, dispares en términos de los criterios que los definen, y variados con respecto a los miembros que los integran, aunque la diferencia evidentemente puede haberse intensificado durante la transición demográfica urbana. Sin embargo, también es innegable que, con suma frecuencia, los hogares funcionan como ámbitos práctico-materiales, por no mencionar que sirven como conducto de ideologías e instituciones más amplias sobre familia y parentesco, dentro de las cuales el género ocupa un papel central (Barrett, 1986; Kuznesof, 1989:169). El hogar también se ha descrito como un órgano con poder de generación considerable. Según Netting, Wilk y Arnould (1984:xxii), se trata de un campo donde "la materia prima de la cultura se media y transforma en acción". Si se reconoce que el hogar es la institución que afecta de manera más inmediata el comportamiento, la identidad y la capacidad de los hombres y las mujeres para determinar sus propias vidas, no es de sorprender que la investigación realizada desde una perspectiva de género y/o feminista, haya empezado a considerar el análisis de esta institución, desde diferentes puntos de vista, como fundamental para obtener una comprensión teórica más matizada de la migración selectiva por género, en particular bajo los auspicios de un "enfoque de estrategias de hogares" para la movilidad demográfica.

\section{El "enfoque de las estrategias de bogares" en la migración por género}

Este "enfoque" para la migración por género, que se articuló como tal en el trabajo de Sarah Radcliffe (1996 y 1991), se derivó de la insatisfacción con la manera en que las teorías neoclásicas y marxistas convencionales sobre movilidad rural-urbana examinaban las relaciones sociales dentro de los hogares. Este enfoque estudia, en cambio, las percepciones derivadas del análisis del comportamiento que toma en consideración los componentes ideológicos y culturales que influyen sobre las reacciones de hombres y mujeres ante el cambio en las condiciones socioeconómicas y ambientalés. Una de las preocupaciones principales del "enfoque de estrategias de hogares" consiste en cuestionar el poder dentro de las unidades, y examinar cómo afecta éste la propensión y la libertad de los diferentes individuos, según su género, edad, relación con otros 
miembros del hogar, etcétera, para participar en la migración hacia las ciudades.

Las consideraciones anteriores resultan vitales cuando los hombres y las mujeres evidentemente no constituyen grupos separados cuyo movimiento a través del espacio coincide limpia y uniformemente con los diferenciales en cuanto a oportunidades de empleo y/o salarios entre campo y ciudad. Por ejemplo, aunque es más probable que las mujeres se desplacen hacia las ciudades si su participación en la agricultura es limitada, tal participación la determinan factores como el género y la división generacional del trabajo de los hogares rurales. Por otra parte, las ideologías sobre la familia, el parentesco y el género, desempeñan un papel importante en los motivos para emigrar y en la autonomía relativa para la toma de decisiones por parte de los emigrantes (Chant, 1991; Chant y Radcliffe, 1992). Aunque el ingreso bien puede ser una razón importante para la migración femenil, por ejemplo, debe reconocerse que las mujeres tal vez no se desplacen hacia las ciudades por voluntad propia, sino por mandato o presiones de sus familias rurales. En el sureste asiático, por ejemplo, con frecuencia se ejerce una presión considerable sobre las hijas solteras, tanto adolescentes como adultas, para que "recompensen" a sus padres por su crianza. Aunque es posible que también se instilen nociones similares de deber y devoción filial en los hijos varones, la evidencia de países como Filipinas, Taiwán e Indonesia sugiere que los padres generalmente prefieren que sean las hijas quienes se conviertan en emigrantes laborales, porque es más probable que éstas envíen dinero a casa (Chant y Mcllwaine, 1995; Mather, 1988; Salaff, 1990; Wolf, 1990). En otros contextos, como el sur de Asia, las ideologías prevalecientes sobre género pueden inhibir cualquier movilidad independiente por parte de las mujeres. Aunque la investigación reciente sobre Bangladesh sugiere que la expansión del empleo femenil en las fábricas de las áreas urbanas está alentando las corrientes de mujeres emigrantes solas que se dirigen a las ciudades (Kabeer, 1991:257), en la mayor parte de la región las limitaciones sociales y culturales que se imponen o filtran por medio del hogar, generalmente han confinado la migración a desplazamientos para contraer matrimonio o para unirse a los esposos que ya se han establecido en los pueblos o ciudades (Oberai y Singh, 1983; Thadani y Todaro, 1984). En América Latina, por el contrario, la evidencia sobre México y Costa Rica sugiere que las mujeres de áreas rurales pueden emigrar para escapar del matrimonio cuando las relaciones con los cónyuges son violentas o de explotación, o para liberarse de los lazos de parentesco que sofocan su libertad o autonomía (Chant, 1996b: cap. 6).

Si bien lo anterior ofrece una visión sobre las diferentes maneras en que la dinámica interna de los hogares puede dar forma a la migración selectiva por género, también es importante tomar en cuenta que existen variaciones considerables en cuanto a la forma en que están constituidos los hogares de las áreas rurales. Estas variaciones contribuyen a mediar el movimiento selectivo por género. Si se acepta que es imposible generalizar los efectos de la estructura de los hogares sobre el género según edad, "raza", divisiones culturales, etcétera (McIlwaine, 1993; Trotz, 1996), en uno de los extremos del espectro, los miembros de aquellos hogares cuyos "jefes" varones ${ }^{6}$ han emigrado a las áreas urbanas o al extranjero, se caracterizan por su dependencia de las remesas y, como resultado, permanecen en las áreas rurales. Por el contrario, a las mujeres que enviudan o se separan de sus cónyuges, no les queda más opción que desplazarse hacia las áreas urbanas si no tienen acceso a un medio de vida en el campo. El acceso a un medio de vida puede verse limitado si las mujeres no cuentan con derechos propios para poseer tierras, si carecen de la mano de obra y/o los recursos financieros para cultivarlas, o si se ven socialmente impedidas para hacerlo (véanse, por ejemplo: Bradshaw, 1996, sobre el caso de Honduras; Drèze, 1990, sobre India; Radcliffe, 1996,

${ }^{6}$ El término "jefe de hogar ${ }^{\mathrm{n}}$ resulta extremadamente problemático, ya que asume la existencia de una persona dentro de la unidad residencial que es *responsable ${ }^{\mathrm{m}}$ de los demás miembros, y/o que está "a cargon de la organización del hogar. En términos generales, en los casos en que el hogar incluye hombres adultos, los encargados del levantamiento censal, las oficinas de gobierno, y los propios miembros del hogar. casi siempre designan como jefe de hogar a una persona de género masculino. La asociación persistente entre género masculino y jefe de hogar se deriva de factores tales como la primacía generalizada que se concede a los hombres dentro de las redes de parentesco, la exportación de ideales eurocéntricos sobre la organización de la familia durante el proceso de colonización, y el papel acostumbrado de los hombres como proveedores del hogar y/o árbitros principales en la toma de decisiones hombres como proveedores del hogar y/o arbitros principales en la toma de decisiones (Folbre, 1991; Harris, 1981). Dicho lo anterior, lo que la jefatura de hogar implica realmente en la práctica rara vez se somete a escrutinio (Stolcke, 1992:138n) y, como ha indicado Jeanne Illo (1992:182), la referencia constante a un solo jefe de hogar, en especial de género masculino, no sólo es señal de la complejidad de los sistemas de asignación que rigen en los hogares, sino que refuerza el poder del varón dentro de la sociedad en su conjunto. En realidad, en la mayoría de los censos en que se registra a las mujeres como jefas de hogar aparecen sólo en los casos en que no existe un compañero u otro hombre "importanten dentro del hogar, como pudiera ser un padre o hermano (UN, 1991:17). También es usual que los censos registren aquellas unidades con jefaturas femeninas de facto como si estuvieran encabezadas por hombres, a menos que los emigrantes hayan estado ausentes del hogar por un periodo de tiempo sustancial, como por ejemplo un año. Para una discusión más detallada, véase Chant, 1996b, pp. 5-10 
sobre Perú; Rahat, 1986, sobre Paquistán). A su vez, esto puede conducir a pérdidas en la productividad rural, degradación ambiental y una mayor pobreza de las comunidades rurales (Chant, 199220). Esto Último revela no sólo la manera en que las formas particulares de composición de los hogares pueden influir sobre la acción personal, sino aquella en que las normas para la organización de los hogares pueden plantear dificultades a los individuos, quienes por una u otra razón, finalmente optan por otras soluciones. A su vez, esto tiene implicaciones más amplias para el cambio y la sustentabilidad del desarrollo, y también resulta relevante para las áreas urbanas.

\section{Diversidad de los bogares en las áreas urbanas}

Un cuerpo cada vez mayor de investigación empírica demuestra que en los hogares de las áreas urbanas de las regiones en desarrollo no predomina, en modo alguno, el "estereotipo ideal" de la familia nuclear con jefatura masculina. Aunque en algunas ciudades siguen prevaleciendo los hogares nucleares, abundan las variaciones en cuanto a tiempo y espacio (Brydon y Chant, 1989: cap. 6; Beall, 1996a; Moore, 1994). Por ejemplo, hasta 50\% de los hogares considerados en las encuestas levantadas en áreas urbanas de Visayas, en Filipinas, son unidades extensas y complejas que incluyen a parientes, empleados domésticos o aprendices, personas que viven solas, personas que viven con amigos, hogares donde los jefes son los abuelos; y allí, al igual que en otras partes del mundo en desarrollo, una minoría significativa de unidades domésticas son encabezadas por mujeres (Chant y Mcllwaine, 1995: cap. 3).

En el sureste asiático, así como en América Latina y el Caribe, los hogares con jefatura femenina tienden a ser un fenómeno más urbano que rural, lo cual refleja un sesgo femenino en los flujos migratorios y una consecuente feminización de la proporción por género en la mayoría de las áreas urbanas. Sin embargo, como se dijo antes con relación a los flujos migratorios, los factores demográficos (o económicos) por sí mismos tienen un poder explicativo limitado cuando se les despoja de sus corolarios sociales. En la misma medida en que los hogares funcionan como escenarios, filtros y generadores de relaciones e ideologías particulares, el poder que tienen estas últimas en otros terrenos (parentesco, religión, mercado de trabajo, Estado, y demás) también ejerce un impacto en la formación de los hogares. Las cuestiones que tienen mayor influencia sobre los niveles de jefatura femenina en diferentes áreas, por ejemplo, incluyen una serie compleja e interactiva de factores demográficos, económicos, legales-institucionales y socioculturales, que van desde diferencias entre hombres y mujeres en cuanto a la edad para contraer matrimonio, a la esperanza de vida según género, al comportamiento de la fecundidad, a los derechos de propiedad sobre la tierra y otros bienes, a las actitudes e intervenciones del Estado (leyes sobre familia y divorcio, programas de bienestar social, etcétera), a los roles de género, relaciones, desigualdades e identidades, sistemas de parentesco, prácticas matrimoniales, y códigos morales y sexuales (para una descripción más detallada véanse: Chant, 1996b: cap. 4; Baylies, 1996; Folbre, 1991; Moore, 1996). En algunos contextos, la jefatura femenina prospectiva es mayor que su incidencia real. Esto se debe a que, por razones económicas, sociales o ideológicas, las unidades integradas por madre e hijo no pueden establecerse de manera independiente y viven como "subfamilias" dentro de hogares extensos más grandes. Por ejemplo, mientras que algo más de dos terceras partes de las madres solteras de las comunidades que incluyeron los estudios de caso en México y Costa Rica encabezan sus propios hogares, esto corresponde a menos de $50 \%$ de los casos que se registraron en Filipinas. Aparentemente, factores tales como la feminización de las proporciones por género en las áreas urbanas y los niveles elevados de participación de la mano de obra femeniná, permitirían que las mujeres filipinas encabezaran sus propios hogares; pero esto se ve contrarrestado por la reprobación social respecto a que las mujeres vivan solas, a la ilegalidad del divorcio, a la relevancia que tienen para la sociedad filipina la armonía familiar y las "buenas relaciones interpersonales", a la importancia que la cultura filipina atribuye a los hijos, y al énfasis en la solvencia moral de las mujeres (en especial en centros de comercio sexual reconocidos). De esta manera, para disimular su situación, acallar las murmuraciones y evitar la vergüenza para sus familias, las jóvenes filipinas que conciben hijos fuera del matrimonio o que se enfrentan a la disolución de la pareja, son absorbidas por los hogares de los padres u otros familiares (Chant, 1996b: cap. 6; también Drèze, 1990, y Vera-Sanso, 1994 para el caso de India). Mientras que a ciertas mujeres reconocidas como jefas de hogar las sostienen sus familiares, otras hacen una contribución económica sustantiva, si bien no la principal, al hogar (Chant y Mcllwaine, 1995). Esto también parece estar aumentando en los hogares con jefes varones en diferentes partes del mundo en desarrollo, y ha dado como resultado que se otorgue mayor 
importancia al hecho de reconocer los hogares "sostenidos por mujeres" (Beall, 1996b; Moore, 1996). A pesar de que sigue estando firmemente arraigado el "mito de que el arreglo en que el hombre es quien gana el pan y la mujer es el ama de casa es normal y mejor para los seres humanos" (Cleves Mosse, 1993:37), los nuevos datos han atraído la atención hacia la división del trabajo dentro de los hogares, y a la forma como los patrones diferenciados podrían (o de hecho deberían) afectar la estructura futura de las intervenciones en materia de bienestar social.

Examinar lo que ocurre dentro de los hogares también puede poner en tela de juicio las percepciones que se tienen acerca de la pobreza. En realidad, el hecho de que la jefatura femenina se haya calificado convencionalmente como causa y consecuencia de la pobreza, en parte se deriva de la falta de problematización de las relaciones que se dan dentro de los hogares, aunque las conceptuaciones deficientes de la pobreza también han tenido que ver en esto.

\section{La pobreza}

La complejidad de la pobreza se ha ocultado convencionalmente a causa de definiciones y técnicas de medición poco refinadas. El uso común de las líneas de pobreza que se basan en el ingreso, según las cuales se define a los pobres como aquellos cuyos ingresos son insuficientes para solventar las necesidades básicas,' ofrece una visión inacabada de las causas subyacentes a la pobreza. Por otra parte, los ingresos por sí solos dicen muy poco sobre las dimensiones sociales de la carencia, o de cuestiones relacionadas con el bienestar. Los ingresos bajos, por ejemplo, pueden no ser particularmente problemáticos si las personas viven en lugares adecuados y tienen acceso a los servicios y a la atención médica necesarios, o si cuentan con una base sólida de bienes, ya sean económicos o físicos (mano de obra, ahorros y recursos), o bien, sociales (educación, capacitación o poder para solicitar ayuda a grupos familiares en tiempos de necesidad) (Beall, 1996b; Chambers, 1995; Mcllwaine, 1996; Moser y McIlwaine, 1995; Wratten, 1995).

' Es evidente que existen diversos tipos de líneas de pobreza basadas en el ingreso. Algunas se basan en una "canasta básica" de alimentos esenciales; otras incluyen el acceso a los servicios y otros factores. También es importantetener en cuenta que, en este caso, el ingreso se refiere al manejo de los recursos a lo largo del tiempo,o al consumo que es posible cuando el capital permanece intacto (Wratten, 1995:12).

En años recientes, las consideraciones anteriores han servido de punto de partida a los intentos de crear bases más sólidas para evaluar y comprender la pobreza, en especial a la luz de un aumento relativo de su expansión y visibilidad en el ámbito urbano en comparación con el rural. Las razones a que se atribuye la mayor incidencia de la pobreza urbana casi siempre han puesto énfasis en la instrumentación de los programas de ajuste estructural (PAE) Éstos han provocado una reducción de los salarios y el empleo en poblados y ciudades por medio de recortes en el gasto público y en las burocracias gubernamentales (UNCHS, 1996:117). Los PAE también han promovido la agricultura de exportación, que al combinarse con una disminución en los subsidios a los alimentos básicos, han tendido a favorecer a los habitantes rurales en mayor grado que a los urbanos; esto debido principalmente a la capacidad que tienen los primeros para autoabastecerse con la agricultura de subsistencia, que ha ayudado a amortiguar el alza en los precios al consumidor (Demery et al., 1993:3-4, y Baden, 1993:4; Afshar y Dennis, 1992, y Asthana, 1994, hacen comentarios en contra). Sin embargo, otro factor que se ha considerado importante para cerrar la brecha entre pobreza rural y urbana, es el aumento en la concentración de la población en las áreas urbanas a lo largo del tiempo. Esto, entre otras cosas, ha transferido la pobreza a los pueblos y ciudades, y ha exacerbado la presión sobre la vivienda y los mercados de trabajo urbanos (Amis, 1995:146; Wratten, 1995). No obstante las dificultades para conceptuar la pobreza urbana de manera distinta a la pobreza rural (Wratten, 1995), entre los factores que según la UNCHS (1996:111) exponen a los habitantes urbanos a un mayor riesgo de pobreza, están: los costos de vida más elevados; mayor dependencia de los ingresos monetarios; una base más reducida de "bienes intangibles", tales como las redes de parentesco; y una mayor exposición a los riesgos ambientales y de salud derivados de una densidad poblacional más elevada, de la provisión inadecuada de servicios de drenaje, alcantarillado y desecho de desperdicios, y de la proximidad a las industrias contaminantes (Beall, 1996b:433; Satterthwaite, 1995).

Entre las iniciativas recientes para evaluar la pobreza destacan las definiciones que se basan en indicadores sociales y económicos en los que la información sobre ingresos se complementa con datos sobre la esperanza de vida, la alfabetización, el acceso a la atención de la salud, etcétera. Otro adelanto es el uso de definiciones participativas en las que la percepción individual de la pobreza se entreteje con el análisis (Wratten, 1995:15 y ss.). Esto 
último ha dado lugar a conceptos nuevos, tales como "vulnerabilidad", que se refiere a la "exposición a la indefensión". Según este concepto, los hogares "vulnerables" son aquellos proclives a sufrir impactos, tensión y riesgo, cuyas respuestas a estas eventualidades implican un desgaste grave de sus bienes (Beall, 1996b:430; Chambers, 1995:175). Los conceptos de Amartya Sen sobre los derechos y capacidades han sido cruciales para una comprensión más profunda de la pobreza y la vulnerabilidad (Sen, 1981, 1985 y 1987a), y han servido para apoyar la observación de que la vulnerabilidad implica reconocer no sólo cómo los pobres obtienen recursos y derechos, sino también cómo los manejan (Mcllwaine, 1996; Moser, 1996).

\section{Pobreza intrabogares y oulnerabilidad}

Conocer la forma en que se distribuyen los recursos dentro del hogar, es crucial para el análisis de la vulnerabilidad. El interés en este tema ha estado acompañado ( $y$ conformado) por cambios más amplios del modelo dentro del campo de la economía de género y de hogares. Estos cambios abarcan desde la "nueva economía de los hogares" ligada al trabajo de Gary Becker, hasta la "nueva economía institucional" que se relaciona con la investigación de Amartya Sen (1987b y 1990) y su modelo de "conflicto de cooperación" (Kabeer, 1994a: cap. 5). Mientras que la "nueva economía de los hogares" trata a éstos como unidades unificadas en donde el ingreso pasa a formar parte de una bolsa común y el trabajo se asigna de conformidad con los principios de la ventaja comparativa (Koopman, 1991:148), la "nueva economía institucional", de acuerdo con el enfoque de "las estrategias de hogares" sobre la migración, adopta una visión que involucra una mayor complejidad de las relaciones que se dan dentro del hogar. Según este esquema, se reconoce que las relaciones de poder y jerarquía son una característica de la vida familiar, tanto como lo es la cooperación, y en oposición a las nociones de altruismo que propone el modelo de Becker, los hogares podrían describirse con mayor precisión como "conjuntos incómodos de estrategias individuales de sobrevivencia" (Bruce y Dwyer, 1988:8; Schmink, 1984), o como ha dicho Moore (1994:87), "un ámbito donde los intereses, los derechos, las obligaciones y los recursos entran en competencia".

La divisa teórica de la nueva economía institucional se ha visto superada por una cantidad cada vez mayor de evidencia empírica que demuestra que dentro de los hogares, las mujeres y las

niñas con frecuencia se encuentran en seria desventaja con relación a ingreso, consumo, nutrición, acceso a la educación, atención de la salud, etcétera (Chant, 1996b: cap. 2). Asimismo, la exposición a presiones tales como la falta de poder personal sobre los recursos, la sujeción a la violencia doméstica, y otros factores, contribuyen a la enorme vulnerabilidad de las mujeres. Debido a que éstas sólo pueden evadir tales dificultades abandonando los hogares encabezados por hombres y estableciéndose por su cuenta, la premisa que se acepta de manera convencional, referente a que los hogares con jefatura femenina son los "más pobres entre los pobres" (Badshah, 1996:35; INSTRAw, 1992:237; Tinker, 1990:5; UNDAW, 1991), podría quedar algo fuera de lugar., La pobreza involucra bastante más que los ingresos y las posesiones materiales, y tiene que ver con el poder, el control, los derechos y las cuestiones de distribución.

\section{Hogares con jefatura femenina: dilemas y descripciones}

Lo anterior no quiere decir, sin embargo, que la discriminación contra las mujeres en general, y contra los hogares con jefatura femenina en particular, facilite que las mujeres tengan un medio de vida decente o sustentable en las ciudades de la mayoría de los países en desarrollo (Moore, 1996:62). A las mujeres se les discrimina no sólo dentro del mercado de trabajo, sino también en otras esferas de la sociedad. A pesar de que la jefatura femenina rara vez representa una "primera opción" para las mujeres, y de que la mayoría de ellas accede a esta condición debido a que los hombres se oponen a contraer matrimonio, al abandono de sus compañeros o a la viudez, la idea popular que se tiene de los hogares con jefatura femenina es que son, en el mejor de los casos, entidades desventuradas y vulnerables $y$, en el peor, ejemplos de reproducción de la patología social, en especial si las mujeres no están casadas (Chant, 1996b; Duncan y Edwards, 1996; McIntosh, 1996; Phoenix, 1996). Aunque los hogares con jefatura femenina (en particular aquellos donde la jefa es una madre soltera o viuda) ${ }^{8}$ en algunas ocasiones son objeto de los programas de combate a la pobreza o de proyectos de

${ }^{8}$ La brevedad del presente trabajo no permitió una discusión del inmenso rango de jefas de hooar que exiscentero de las sociedades en desarrollo. En Chant, 1996b, pp. 10-26, 
bienestar, por considerarse que se trata de grupos "vulnerables", es menos probable que la política social o familiar de la tendencia principal piense en ellos como grupos integrales, por no mencionar las iniciativas de desarrollo a nivel macro. ${ }^{10}$ Los estados realizan pocos esfuerzos por apoyar a los hogares con jefatura femenina, $y / o$ socavan indirectamente su legitimidad al promover el ideal de la familia con un padre y una madre, y al formular la política social alrededor de este modelo. Las acciones gubernamentales normalmente reciben apoyo de otras autoridades - tales como grupos religiosos-, con las que se combinan para marginar y excluir a las jefas de hogar de posiciones sociales que les permitan mejorar su bienestar financiero. En realidad, estas influencias se filtran hasta el ámbito de la comunidad y hacen que la jefatura femenina sea más difícil de lo que podría ser (Chant, 1996b: caps. 7 y 8; Lewis, 1993). En vista del aumento en el número de hogares con jefatura femenina en la mayoría de las sociedades en desarrollo, las limitaciones referidas no parecerían ser una base particularmente óptima sobre la cual fundar un desarrollo participativo y sustentable. De hecho, este grupo parecería estar particularmente sujeto a la "exclusión social" que tantos protagonistas del desarrollo sustentable desean evitar. ${ }^{11}$

9 A pesar de que un número cada vez mayor de gobiernos de países en desarrollo está incluyendo a los hogares con jefatura femenina en los programas de pobreza [como por ejemplo Colombia, Chile, Honduras, Costa Rica, Barbados, India y Bangladesh (Beall, 1996a:23; Chant, 1996b: caps. 2 y 5; Grosh, 1994; Lewis, 1993)], con frecuencia estos programas son limitados y problemáticos. Por ejemplo, en Bangladesh la única ayuda a que tienen derecho las jefas de hogar (oficialmente reconocidas como "mujeres pobres indigentes") es el Programa de Alimentación para Grupos Vulnerables (Vulnerable Group Feeding Programme) y el Programa de Alimentación para el Trabajo (Food for Work Programme). Como indica David Lewis (1993:33), ambos programas están "más cercanos a la asistencia social que al desarrollo en cuanto a su orientación, y conllevan limitaciones a insustentabilidad además del problema moral de utilizar a la pobreza como fuente barata de mano de obra".

${ }^{10}$ Esto encuentra paralelo en el trabajo de Marty Chen (1989) sobre género y participación conómica, donde se indica que las mujeres con frecuencia son meta de los programes basados en la comúnidad y la antipobreza que bien podría incluir proyectos lonerogramas basados de pequeña escala pero se les excluye le plancios organizarse de mar manera sectorial, más que espacial y que se orienta al desarrollo $y$ al crecimiento

11" "Exclusión social" es un concepto que describe la exclusión de los mercados de trabajo y de los derechos cívicos y políticos, y tal como lo indica la UNCHS (1996:116), ha tenido resonancia particular dentro de los debates sobre la "nueva pobreza" en Europa, generada a raíz del cambio tecnológico y la restructuración económica. Sin embargo, el concepto se usa con jefatura femenina, sino con respecto a los mercados de trabajo informales (Beall, 1996c).

\section{Dinámica y diversidad de los bogares: el reto para las políticas}

El objetivo en este trabajo ha sido llamar la atención hacia la importancia que tienen las instituciones sociales para la planeación del desarrollo sustentable y, más específicamente, hacia la necesidad de examinar al "hogar" a la luz de las políticas orientadas a abordar los conflictos de género, el desarrollo urbano, y el bienestar de los pobres de las áreas urbanas. Los hogares se caracterizan por presentar diferencias considerables, que reciben influencia tanto de las acciones de los individuos que los integran, como de ideologías y grupos más amplios. La organización de los hogares también ha sido modelada directa o indirectamente por medio de las leyes, la planeación y las acciones de los gabiernos y los grupos hegemónicos durante el proceso de desarrollo. Carolyn Baylies (1996:87), por ejemplo, señala la situación contradictoria con la que "el desarrollo capitalista simultáneamente alienta la familia nuclear y crea las condiciones para su disolución". También indica que "el poder de la imaginación colectiva" (ibidem:77) sobre los nexos entre el hogar nuclear y la modernización es tal, que incluso cuando las influencias han sido directas, éstas rara vez se han hecho explícitas. ${ }^{12}$ Por el contrario, los hogares (y las familias) con jefatura masculina se han convertido en entidades "naturales". Esto es importante porque, como señala Naila Kabeer (1994b:889) con respecto a las relaciones de género:

debido a que las ideologías naturalistas han resultado más eficaces para ocultar la base social de las relaciones de género que la mayoría de las formas de desigualdad, y dado que las implicaciones de las formas de desigualdad basadas en el género se experimentan dentro de los terrenos más íntimos $\mathrm{y}$ personalizados de nuestra existencia, con frecuencia es difícil darse cuenta de que las relaciones de género son un producto social con historia. ${ }^{13}$

12 Éste es menos el caso de las economías avanzadas, como la del Reino Unido, donde las iniciativas que el Partido Conservador propuso en los años noventa hicieron ampliamente explícita su adherencia a la norma de la vida familiar "tradicional" de padre y madre (Duncan y Edwards, 1996; Rosenheil y Mann, 1996)

${ }^{13} \mathrm{El}$ argumento de Kabeer hace eco en Eva Rathgeber (1995:207), quien añade que "se considera que las relaciones sociales de género caen dentro del terreno de la cultura, y que $h$ defensa decidida de repensar las relaciones de género se consideraría como una 'interferenci cultural' sin bases". 
De manera más insidiosa, el hecho de culpar a las familias, y con suma frecuencia a las mujeres dentro de éstas, por su "incapacidad para sobrevivir a los cambios estructurales que ha generado una mayor integración del mercado, es una manera de evitar el análisis de las causas y las consecuencias de la pobreza y la miseria" (Moore, 1996:74). Utilizar a los hogares "irregulares" como chivos expiatorios también puede reforzar ideales sobre la maternidad dentro del matrimonio que pueden resultar adversos para las mujeres, por no mencionar el progreso hacia una igualdad entre géneros (McIntosh, 1996; Rosenheil y Mann, 1996).

Si bien las intervenciones legislativas y de desarrollo han formado parte de la historia de la organización de los hogares hasta nuestros días, no existe nada que pueda detener su continuidad. En realidad, esto podría ser altamente deseable siempre y cuando existiera una mayor aceptación y sensibilidad en torno a las cuestiones de la diferencia y la diversidad, y para garantizar que las mujeres dentro de los hogares obtuvieran un trato más justo que el que reciben en la actualidad. La inversión en los individuos (y aquí debe reconocerse que un gran número de jefas de familia tienen hijos, al igual que un número extenso de parejas en unidades con jefatura masculina) es una de las piedras angulares del desarrollo sustentable, y equivale a una "estrategia para salvaguardar el potencial productivo del futuro" (Moore, 1996:74). Debe reconocerse que aunque los hogares con jefatura femenina pueden ser "diferentes" (o cuando menos distintos a lo que establece la norma de la corriente principal), de ninguna manera están "desviados" o son "disfuncionales". Como ha argumentado Jo Beall (1996a:25) con relación a la forma de gobierno urbana: "Para que el compromiso cívico aproveche totalmente la combinación de energía y creatividad humanas, las ciudades deben ser inclusivas y acoger la diversidad social". En vista de la enorme importancia que tiene el hecho de conducir a las personas de las bases a realizar movimientos para el desarrollo sustentable, la aprobación y el apoyo institucionales a nivel macro de la pluralidad en cuanto a tipos de hogar representarían un paso significativo en esa dirección.

Las medidas prácticas que pueden poner en marcha los Estados, las agencias internacionales y las ONG para facilitar, si no para fomentar, la diversidad de los hogares y hacerlos lugares más justos para sus miembros, podrían incluir iniciativas para mejorar las relaciones de género dentro de estas unidades, poniendo énfasis en la responsabilidad compartida por aquellas tareas que recaen exclusivamente en las mujeres (tales como el trabajo doméstico y el

cuidado infantil), reconociendo el papel del padre mediante cambios en el lugar de trabajo (licencias de paternidad, guarderías, etcétera), haciendo que existan facilidades para el cuidado de los niños y que sean accesibles para todos los padres y madres, y garantizando que los programas de vivienda incluyan a los hogares de diferentes tipos. ${ }^{14} \mathrm{El}$ hecho de que las mujeres jefas de hogar deban o no ser una meta específica, es causa de cierto debate. Entre los encargados de la formulación de políticas, en particular, el temor a que el hecho de apoyar a las madres solteras pueda exacerbar este síndrome ha significado que, hasta ahora, pocos países se hayan aventurado a transitar por este camino (Beall, 1996a:23; Chant, 1996b: cap. 2). El punto crucial, sin duda, es empezar a preguntar y a escuchar las voces de quienes, desde las bases, realmente experimentan el "problema", y cuyo futuro probablemente dependerá de intervenciones de políticas adecuadas.

\section{Bibliografía}

Afshar, Haleh y Carolyne Dennis (1992), "Women, Recession and Adjustment in the Third World: Some Introductory Remarks", en Halh Afshar y Carolyne Dennis (eds.), Women and Adjustment Policies in the Third World, Basingstoke, Macmillan.

Amis, Philip (1995), "Making Sense of Urban Poverty", Environment and Urbanization, vol. 7, núm. 1, pp. 145-147.

Asthana, Sheena (1994), "Economic Crisis and the Impact on Health", en David Phillips y Yola Verhasselt (eds.), Health and Development, Londres, Routledge.

Baden, Sally (1993), The Impact of Recession and Adjustment on Women's Work in Developing Countries, Sussex, IDS, Bridge Report núm. 15.

${ }^{14}$ En su reseña sobre género y gobernabilidad urbana (en la cual defiende tanto la plena participación de las mujeres en el desarrollo de los asentamientos humanos, como una mayor conciencia de género entre hombres y mujeres dentro de la vida folíica y la práctica de planeación), Jo Beall (1996a:23) indica cómo el desarrollo - por parte del gobierno sueco- de planeacion), Jo Beall (19i:2 ) indica cóno el desarollo por parte del gobierno suecoparto 
Badshah, Akhtar (1996), Our Urban Future: New Paradigms for Equity and Sustainability, Londres, Zed.

Barret, Michèle (1986), Women's Oppression Today: Problems in Marxist Feminist Analysis, Londres, Verso.

Baylies, Carolyn (1996), "Diversity in Patterns of Parenting and Household Formation", en Elizabeth Bortolaia Silva (ed.), Good Enough Mothering? Feminist Perspectives on Lone Motherbood, Londres, Routledge.

Beall, Jo (1996a), Urban Governance: Why Gender Matters, Nueva York, UNDP, Gender in Development Monograph Series, núm. 1

(1996b), "Social Security and Social Networks Among the Urban Poor in Pakistan", Habitat International, vol. 19, núm. 4, pp. 427-445.

(1996c), "Households, Livelihood and the Urban Environment: Social Development Perspectives on Solid Waste Disposal in Faisalabad City, Pakistan", Londres, Departamento de Geografía/London School of Economics, tesis de doctorado (en elaboración).

Bradshaw, Sarah (1996), "Female-headed Households in Honduras A Study of Their Formation and Survival in Low-income Communities", Londres, Departamento de Geografíal London School of Economics, tesis de doctorado.

Bruce Judith y Daisy Dwyer (1988), "Introduction", en Daisy Dwyer y Judith Bruce (eds.), A Home Divided: Women and Income in the Third World, Stanford, Stanford University Press.

Brydon, Lynne y Sylvia Chant (1989), Women in the Third World: Gender Issues in Rural and Urban Areas, Aldershot, Edward Elgar.

Chambers, Robert (1995), "Poverty and Livelihoods: Whose Reality Counts?", Environment and Urbanization, vol. 7, núm. 1, pp. 173-204.
Chant, Sylvia (1991), "Gender, Households and Seasonal Migration in Guanacaste, Costa Rica", European Review of Latin American and Caribbean Studies, núm. 50, pp. 51-85.

(1992), "Conclusion: Towards a Framework for the Analysis of Gender-selective Migration", en Sylvia Chant (ed.), Gender and Migration in Developing Countries, Londres, Belhaven.

(1996a), Gender, Urban Development and Housing, Nueva York, UNDP, Publications Series for Habitat II, vol. 2.

- (1996b), Women-beaded Households: Diversity and Dynamics in the Developing World, Basingstoke, Macmillan.

- y Cathy McIlwaine (1995), Women of a Lesser Cost: Female Labour, Foreign Exchange and Pbilippine Development, Londres, Pluto.

y Sarah Radcliffe (1992), "Migration and Development: The Importance of Gender", en Sylvia Chant (ed.), Gender and Migration in Developing Countries, Londres, Belhaven.

y Peter Ward (1987), "Family Structure and Low-Income Housing Policy", Third World Planning Review, vol. 9, núm.4, pp. 5-19.

Chen, Marty (1989), “A Sectoral Approach to Promoting Women's Work: Lessons from India", World Development, vol. 17, núm. 7, pp. 1007-1016.

Cleves Mosse, Julia (1993), Half the World, Half a Chance Oxford, Oxfam.

Demery, Lionel, Marco Ferroni, Christiaan Grootaert y Jorge Wong-Valle (1993), Understanding the Social Effects of Policy Reform, Washington, D. C., Banco Mundial.

Drèze, Jean (1990), Widows in Rural India, Londres, Suntory International Centre for Economics and Related Disciplines/ London School of Economics, Development Economics Research Programme, núm. 26. 
Duncan, Simon y Rosalind Edwards (1996), "Lone Mothers and Paid Work: Neighbourhoods, Local Neighbourhoods and Welfare State Regimes", Social Politics: International Studies in Gender, State and Society, vol. 3, núm. 4.

Folbre, Naricy (1991), "Women on their Own: Global Patterns of Female Headship", en Rita S. Gallin y Ann Ferguson (eds.), The Women and International Development Annual, vol. 2, Boulder, Westview.

(1994), Who Paysfor the Kids? Gender and the Structures of Constraint, Londres, Routledge.

Fonseca, Claudia (1991), "Spouses, Siblings and Sex-linked Bonding: A Look at Kinship Organization in a Brazilian Slum", en Elizabeth Jelin (ed.), Family, Household and Gender Relations in Latin America, Londres, Kegan Paul International, y París, UNESCO.

Gardner, Katy (1995), Global Migrants, Local Lives: Travel and Transformation in Rural Bangladesh, Oxford, Clarendon Press.

Grosh, Margaret (1994), Administering Targeted Social Programs in Latin America: From Platitudes to Practice, Washington D. C., Banco Mundial.

Harris, Olivia (1981), "Households as Natural Units", en Kate Young, Carol Wolkowitz y Roslyn McCullagh (eds.), Of Marriage and the Market, Londres, CSE Books.

Illo, Jeanne (1992), "Who Heads the Household? Women in Households in the Philippines", en K. Saradamoni (ed.), Finding the Housebold: Methodological and Empirical Issues, Nueva Delhi, Sage.

INSTRAW (1992), "Women and the Household", en K. Saradamoni (ed.), Finding the Household: Methodological and Empirical Issues, Nueva Delhi, Sage.
Kabeer, Naila (1991), "Gender Dimensions of Rural Poverty: Analysis from Bangladesh", Journal of Peasant Studies, vol. 18, núm. 2, pp. 241-262.

(1994a), Reversed Realities: Gender Hierarchies in Development Thought, Londres, Verso.

(1994b), Gender Planning in Development Agencies: Meeting the Challenge, Oxford, Oxfam.

Kennedy, Eileen (1994), "Development Policy, Gender of Head of Household, and Nutrition", en Eileen Kennedy y Mercedes González de la Rocha, Poverty and Well-Being in the Household: Case Studies of the Developing World, San Diego, Center for Iberian and Latin American Studies/Universidad de California en San Diego.

Koopman, Jeanne (1991), "Neoclassical Household Models and Modes of Household Production: Problems in the Analysis of African Agricultural Households", Review of Radical Political Economy, vol. 23, núms. 3-4, pp. 148-73.

Kuznesof, Elizabeth (1989), "The History of the Family in Latin America: A Critique of Recent Work", Latin American Research Review, vol. 24, núm. 2, pp. 168-186.

Lewis, David (1993), "Going it Alone: Female-Headed Households, Rights and Resources in Rural Bangladesh", European Journal \& Development Research, vol. 5, núm. 2, pp. 23-42.

Masini, Eleanora Barbieri (1991), "The Household, Gender and Age Project", en Eleanora Massini y Susan Stratigos (eds.), Women, Households and Change, Tokio, United Nations University Press.

Mather, Celia (1988), "Subordination of Women and Lack of Industrial Strife in West Java", en John Taylor y Andrew Turton (eds.), Sociology of "Developing Societies": Southeast Asia, Basingstoke, Macmillan. 
Mcllwaine, Cathy (1993), "Gender, Ethnicity and the Local Labour Market in Limón, Costa Rica", Londres, Departamento de Geografía/London School of Economics, tesis de doctorado.

(1997) "Vulnerable or poor? A study of ethnic and gender disadvantage among Afro-Caribbeans in Limón, Costa Rica", European Journal of Development Research, vol. 9, núm. 2, pp. 35-61.

McIntosh, Mary (1996), "Social Anxieties about Lone Motherhood and Ideologies of the Family: Two Sides of the Same Coin", en Elizabeth Bortolaia Silva (ed.), Good Enough Mothering? Feminist Perspectives on Lone Motherhood, Londres, Routledge.

Miraftab, Faranak (1993), "Shelter as Sustenance: Exclusionary Mechanisms Limiting Women's Access to Housing", en Hemalata Dandekar (ed.), Shelter, Women and Development: First and Third World Perspectives, Ann Arbor, Michigan, George Wahr Publishing Co.

Moore, Henrietta (1994), Is there a Crisis in the Family?, Ginebra, UNRISD, Cumbre Mundial para el Desarrollo, Occasional Paper, núm. 3.

(1996), "Mothering and Social Responsibilities in a Crosscultural Perspective", en Elizabeth Bortolaia Silva (ed.), Good Enough Mothering? Feminist Perspectives on Lone Motherhood, Londres, Routledge.

Moser, Caroline (1996), Confronting Crisis: A Comparative Study of Household Responses in Poverty and Vulnerability in Four Poor Urban Communities, Washington, D.C., Banco Mundial, Environmentally Sustainable Development Studies and Monographs Series, núm. 8.

y Sylvia Chant (1985), The Role of Women in the Execution of Low-income Housing Projects: Training Module, Londres, Development Planning Unit/University College London, Gender and Planning Working Paper, núm. 6. y Cathy Macllwaine (1995), "Poverty and Vulnerability in Commonwealth, Metro Manila, Philippines", Washington, D. C., Banco Mundial, División de Transporte, Agua y Desarrollo Urbano (mimeo.).

Netting, Roben McC., Richard R. Wilk y Eric J. Arnould (1984), "Introduction", en Robert McC Netting, Richard R. Wilk y Eric J. Arnould (eds.), Housebolds: Comparative and Historical Studies of the Domestic Group, Berkeley, University of California Press.

Oberai, A. S. y H. K. Manmoham Singh (1983), Causes and Consequences of Internal Migration: A Study of the Indian Punjab, Nueva Delhi, Oxford University Pras.

Phoenix, Ann (1996), "Social Constructions of Lone Motherhood: A Case of Competing Discourses", en Elizabeth Bortolaia Silva (ed.), Good Enough Motbering? Feminist Perspectives on Lone Motherhood, Londres, Routledge.

Radcliffe, Sarah (1991), "The Role of Gender in Peasant Migration: Conceptual Issues from the Peruvian Andes", Review of Radical Political Economy, vol. 23, núms. 3-4, pp. 148-173.

(1996), "Gender Relations, Peasant LivelihoodStrategies and Migration: A Case Sutdy from Cuzco, Peru", Bulletin of Latin American Research, vol. 5, núm. 2, pp. 29-47.

Rahat, Naveed-I (1986), "Meharabad, A Punjab Village: Male OutMigration and Women's Changing Roles", en Frits Selier y Mehtab Karim (eds.), Migration in Pakistan: Themes and Facts, Lahore, Vanguard.

Rathgeber, Eva (1995), "Gender and Development in Action", en Marianne Marchand y Jane Parpart (eds.), Feminism/ Postmodernism/Development, Londres, Routledge.

Roberts, Penelope (1991), "Anthropological Perspectiva on the Household", IDS Bulletin, vol. 22, núm. 1, pp. 60-64.

Rosenheil, Sasha y Kirk Mann (1996), "Unpalatable Choices and Inadequate Families: Lone Mothers and the Underclass 
Debate", en Elizabeth Bortolaia Silva (ed.), Good Enough Mothering? Feminist Perspectives on Lone Motherhood, Londres, Routledge.

Salaff, Janet (1990), "Women, Family and the State: Hong Kong, Taiwan and Singapore - Newly Industrialized Counties in Asia", en Sharon Stichter y Jane Parpart (eds.), Women, Employment and the Family in the International Division of Labour, Basingstoke, Macmillan.

Satterthwaite, David (1995), "The Underestimation of Urban Poverty and of its Health Consequences", Third World Planning Review, vol. 17, núm. 4, pp. iii-xii.

Schmink, Marianne (1984), "Household Economic Strategies", Latin American Research Review, vol. 19, núm. 3, pp. 87-101.

Sen, Amartya K. (1981), Poverty and Famines, Oxford, Clarendon Press.

(1985), Commodities and Capabilities, Helsinki, United Nations University, World Institute of Development Economics Research.

(1987a), Hunger and Entitlements, Amsterdam, North Holland.

(1987b), Gender and Cooperative Conflicts, Helsinki, United Nations University, World Institute of Development Economics Research, Working Paper, núm. 18.

(1990), "Gender and Cooperative Conflicts", en Irene Tinker (ed.), Persistent Inequalities: Women and World Development, Nueva York, Oxford University Press.

Stolcke, Verena (1992), "The Slavery Period and its Influence on Household Structure and the Family in Jamaica", en Elza Berquó y Peter Xanos (eds.), Family Systems and Cultural Change, Oxford, Clarendon Press.

Thadani, Veena y Michael Todaro (1984), "Female Migration: A Conceptual Framework", en James Fawcett, Siew-Ann Kh y Peter C. Smith (eds.), Women in the Cities of Asia: Migration and Urban Adaptation, Boulder, Westview.

Thorner, Alice y Jyoti Ranadive (1992), "Working Class Women in an Indian Metropolis: A Household Approach", en K. Saradamoni (ed.), Finding the Household: Methodological and Empirical Issues, Nueva Delhi, Sage.

Tinker, Irene (1990), "A Context for the Field and for the Book", en Irene Tinker (ed.), Persistent Inequalities: Women and World Development, Nueva York, Oxford University Press.

Townsend, Janet y Janet Momsen (1987), "Towards a Geography of Gender in the Third World", en Janet Momsen and Janet Townsend (eds.), Geography of Gender in the Third World, Londres, Hutchinson.

Trotz, Alissa (1996), "Gender, Ethnicity and Familial Ideology in Georgetown, Guyana: Household Structure and Female Labour Force Participation Reconsidered", European Journal of Development Research, vol. 8, núm. 1.

United Nations (UN) (1991), The World's Women 1970-1990: Rends and Statistics, Nueva York, UN.

United Nations Centre for Human Settlements (UNCHS) (HABITAT) (1996), An Urbanising World: Global Report on Human Settlements 1996, Oxford, Oxford University Press.

United Nations Division for the Advancement of Women (UNDAW) (1991), "Women and Households in a Changing World", en Eleanora Masini y Susan Stratigos (eds.), Women, Housenolds and Change, Tokio, United Nations University Press.

Vera-Sanso, Penny (1994), "What the Neighbours Say: Gender, Personhood and Power in Two Low-incomeSettlements of Madras", Londres, Departamento de Antropología, Goldsmiths College/Universidad de Londres, tesis de doctorado. 